\title{
Design of a Quasi- elliptic Lowpass Filter using A New Defected Ground Structure and Capacitively Loaded Microstrip Line
}

\author{
Tamasi Moyra ${ }^{1}$, Susanta Kumar Parui ${ }^{2}$, and Santanu Das ${ }^{3}$ \\ ${ }^{1}$ Department of Electronics and Communication Engineering, \\ College of Engineering and Management, Kolaghat \\ ${ }^{2.3}$ Department of Electronics and Telecommunication Engineering, \\ Bengal Engineering and Science University, Shibpur, \\ Howrah - 711 103, INDIA +913326684561/61/63 web: www.becs.ac.in \\ tamasi_moyra@yahoo.co.in, arkapv@yahoo.com \& santanumdas@yahoo.com
}

\begin{abstract}
The modern microwave and millimeter wave communication system demands good filtering characteristics with compact sizes. In this paper the attention has been given towards the design of a good elliptical lowpass filter with sharp transition between passband and stopband, negligible passband insertion loss and wide stop band. A new defected ground structure (DGS) consisting of two square slots connects with a rectangular slot by two thin transverse slots underneath a microstrip line is proposed. In the frequency characteristics of proposed unit pattern provides an attenuation zero close to an attenuation pole. As a result, better transition sharpness, lower passband insertion loss and broader stopband are observed compares to dumbbell DGS. An equivalent lumped L-C network is proposed to model the introduced DGS unit and corresponding L-C parameters are extracted. A 3rd order quasi-elliptic lowpass filter with $1.4 \mathrm{GHz}$ cutoff frequency, $1.7 \mathrm{GHz}$ attenuation pole frequency, negligible passband insertion loss, almost $100 \mathrm{~dB} / \mathrm{GHz}$ sharpness factor and $1.56 \mathrm{GHz}$ passband bandwidth (at $-15 \mathrm{~dB}$ ) is designed by cascading three investigated DGS units of different dimensions under capacitively loaded microstrip line.
\end{abstract}

Keywords: microstrip, defected ground structure, elliptical, lowpass filter, capacitively loaded microstrip line.

\section{Introduction}

A defected structure etched in the metallic ground plane of a microstrip line is attractive solution for achieving finite pass band, rejection band and slow-wave characteristics. The defected structure effectively disturbs the shield current distribution in the ground plane and thus, introduces high line inductance and capacitance of the microstrip line. Thus, it obtains wide stop band and compact size, which meet emerging application challenges. Dumb-bell shaped DGS is explored first time by D. Ahn and applied to design a lowpass filter [1,2]. Unit cell has been described as a one-pole Butterworth filter, where the capacitance comes only from the gap and the inductance comes only from the loop. The study of dumbbell DGS with various head shape have appeared in the literature recently and they are used to design filters, couplers, dividers and amplifiers [3-5] and [7-9]. It is well known that a filter with attenuation poles and attenuation zeros at finite frequencies shows higher selectivity compared to all pole filter. A DGS with quasi-elliptical response was proposed by Chen J.-X recently [6].

In this paper, an asymmetric DGS pattern with reference to transmission line is proposed. Its unit cell consists of two square headed thin slots connected with a rectangular slot under microstrip line transversely. The investigated DGS unit produces an attenuation zero near to the attenuation pole frequency, which yields a very sharp transition band. A general equivalent circuit represented by cauer's $\Pi$-network is proposed for describing the DGS unit. Three DGS cells are cascaded under capacitively loaded microstrip line realize a lowpass filter.

Received: October $2^{\text {nd }}, 2010$. Accepted: January $21^{\text {st }}, 2011$ 


\section{Frequency Characteristics of the DGS}

Figure 1(a) shows the schematic diagram of the investigated DGS unit pattern etched off the backside metallic ground plane underneath a microstrip line. In the layout of DGS unit, a rectangular slot (of length a and breadth $b$ ) is connected with two square slots (of side length e) by two thin transverse slots (of length $c$ and width g). The transverse slots increase the effective capacitance of the microstrip line whereas attached rectangular and square slots increase effective inductance. In order to investigate the frequency characteristics of the DGS unit, it is simulated by the MoM based IE3D EM-simulator. The different dimensions of the DGS unit are considered as $\mathrm{b}=10 \mathrm{~mm}, \mathrm{a}=\mathrm{e}=4 \mathrm{~mm}, \mathrm{~d}=\mathrm{c}=2 \mathrm{~mm}$, and $\mathrm{g}=0.4 \mathrm{~mm}$. The substrate with a dielectric constant of 3.2, loss tangent 0.0025 and thickness $0.79 \mathrm{~mm}$ is considered for the microstrip line. The width $(\mathrm{w})$ of the conductor strip on the top plane is $1.92 \mathrm{~mm}$ corresponding to 50-ohm characteristic impedance.

The prototype structure is fabricated on Arlon PTFE substrate and measured with an Agilent make vector network analyzer of model N5230A. Both the simulated and measured Sparameters are plotted in Figure 1(b). The measured 3-dB cutoff frequency and pole frequency are $3.16 \mathrm{GHz}$ and $3.6 \mathrm{GHz}$, whereas, simulated values are $3.15 \mathrm{GHz}$ and $3.5 \mathrm{GHz}$, respectively. The attenuation zero is observed at $2.83 \mathrm{GHz}$, which is very close to the attenuation pole location $(3.5 \mathrm{GHz})$. As a result, high sharpness factor $(50 \mathrm{~dB} / \mathrm{GHz})$ is obtained in transition band. The passband insertion loss is well below $1 \mathrm{~dB}$. The measured result complies with the simulated result to a great extent.

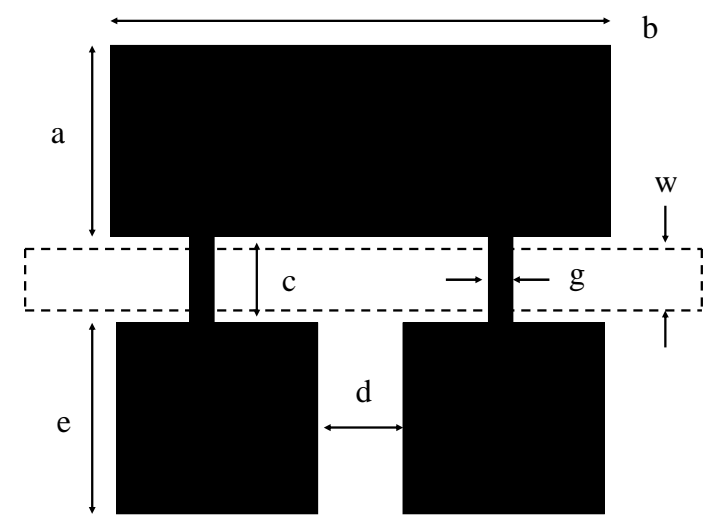

Figure 1(a). Schematic diagram of the proposed DGS unit pattern

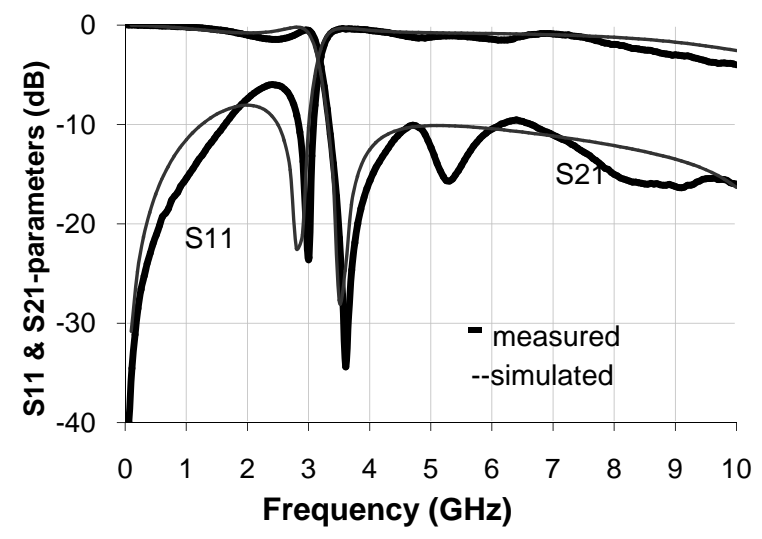

Figure 1(b). Simulated and measured S-parameters 


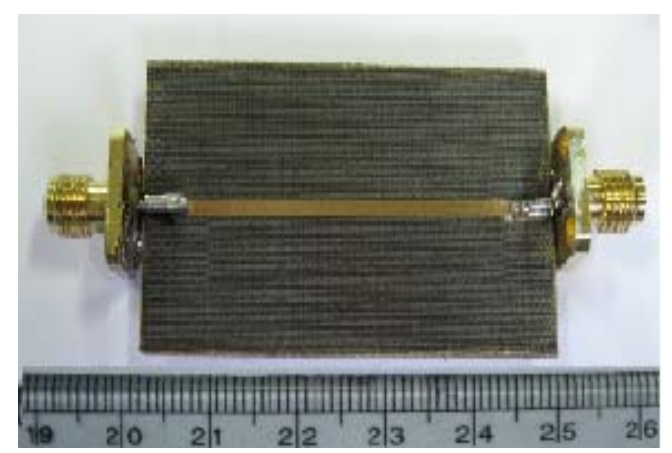

Figure 2(a). Photographic views of signal plane

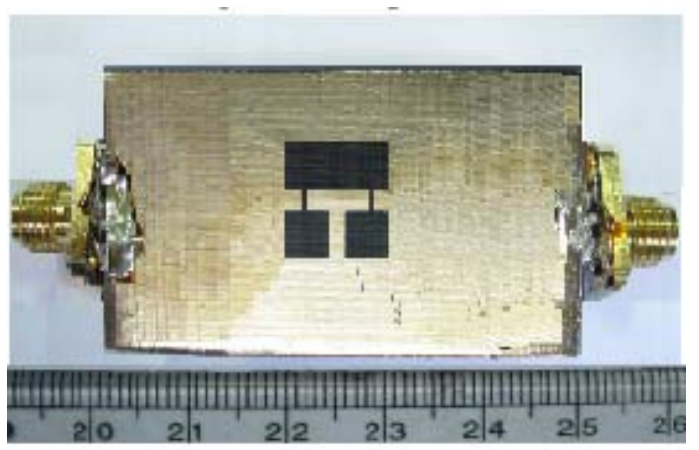

Figure 2(b). Photographic views of ground plane of DGS cell

\section{Equivalent circuit and Parameter Extraction}

Figure 3(b) shows the proposed loss-less equivalent circuit, which is represented by Cauer's $\Pi$-network. The introduced equivalent circuit consisting of series impedance $Y$ a (of $L g, C g$ ) and shunt impedance $Y \mathrm{~b}$ (of $C p$ ) is connected in series with impedance $Z_{0}$ of a transmission line as illustrated in Figure3(b). A DGS unit provides a cut-off and a attenuation pole frequency due to perturbation or disturbance of current distribution in the ground plane. Actually, the transverse slot increases the effective capacitance and the rectangular and square slots area improves the effective series inductance of the transmission line. Thus, the equivalent circuit, the etched defect in the metallic ground plane can be modeled as a parallel LC resonant circuit. But this parallel LC resonant circuit is not sufficient to explain the effect of discontinuities on the performance of the DGS. Here, the equivalent circuit includes the parallel capacitance $C p$ that is due to the relatively large fringing field at the step discontinuity plane on the metallic ground surface.

The simulated S-parameter results are matched to the $3^{\text {rd }}$ order elliptical lowpass filter response having attenuation pole at $3.5 \mathrm{GHz}$, attenuation zero at $2.83 \mathrm{GHz}$ and stopband ratio of 1.1. The equivalent circuit parameters are calculated by using the prototype element values of the $3^{\text {rd }}$ order elliptical lowpass filter. The EM-simulated and circuit-simulated S-parameters are presented in Figure 3(a) and good agreements between them are noticed. The main reason for the discrepancy in attenuation at pole frequency is that ideal model has been adopted in circuit simulation and loss of resister and radiation haven't been taken into consideration. For the dimensions of the DGS unit as mentioned here, we can extract the parameters as $\mathrm{L}_{\mathrm{g}}=1.003$ $\mathrm{nH}, \mathrm{C}_{\mathrm{g}}=2.0481 \mathrm{pF}$ and $\mathrm{C}_{\mathrm{p}}=1.174 \mathrm{pF}$. 


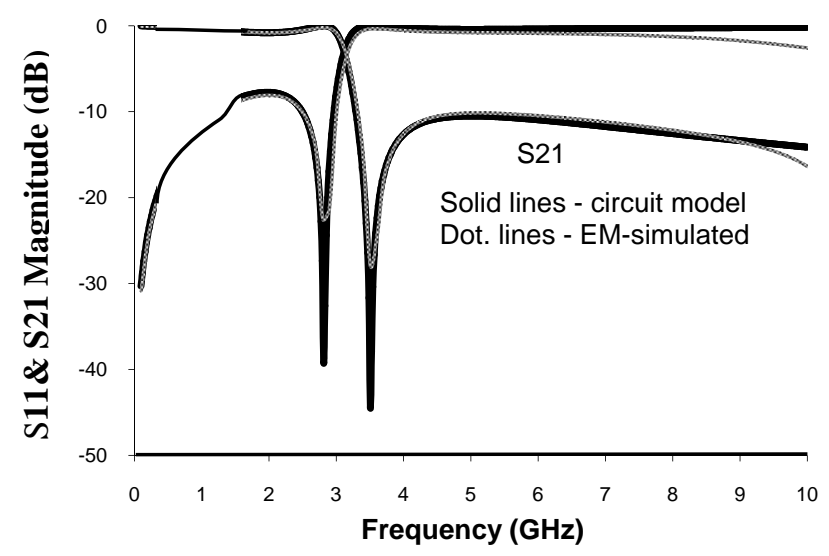

(a)

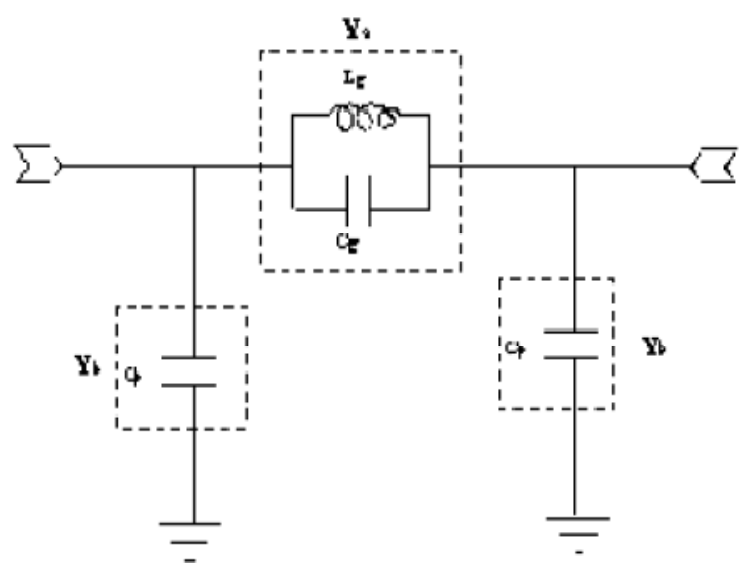

(b)

Figure 3(a). S-parameters of the DGS from circuit-model and EM- simulation (b) Equivalent circuit

\section{Optimization of the DGS shape and Comparison of Proposed DGS unit with Dumbbell DGS}

The elliptic- function response is characterized by the following parameters:

(a) Stopband ratio $\left(r_{s}\right)=f_{s} / f_{p}$ where $f_{s}$ is the stopband edge frequency and $f_{p}$ is the passband edge frequency

(b) Stopband attenuation $\left(\alpha_{\mathrm{s}}\right)$ and

(c) Passband attenuation $\left(\alpha_{p}\right)$.

For almost ideal response, the value of $r_{s}$ should be close to $1, \alpha_{p}$ should be close to zero and $\alpha_{s}$ should be very high. The different dimension of the DGS unit is varied against $r_{s}, \alpha_{p}$ and $\alpha_{\mathrm{s}}$ and obtained a relationship $\mathrm{a}=\mathrm{e} ; \mathrm{d}=\mathrm{c}=\mathrm{e} / 2 ; \mathrm{b}=2 \mathrm{a}+\mathrm{d}=2.5 \mathrm{a}$ for optimum values of $\mathrm{r}_{\mathrm{s}}, \alpha_{\mathrm{p}}$ and $\alpha_{\mathrm{s}}$. It is also observed that $r_{\mathrm{s}}$ increases, i.e., sharpness decreases with increased value of $\mathrm{g}$. So, low value of $g$ is preferred. Finally we reach to a definite shape of the DGS unit with almost ideal elliptic- function response. Now, if any physical dimension (say, b) is known, other dimension (like a, e, d, c) can be determined from these relations. In our earlier example, b is taken as $10 \mathrm{~mm}$ and other dimension are calculated as $\mathrm{a}=4, \mathrm{e}=4 \mathrm{~mm}, \mathrm{~d}=2, \mathrm{c}=2 \mathrm{~mm}$. The value of $\mathrm{g}$ is taken as $0.4 \mathrm{~mm}$. 
It is also possible to design one LPF of almost same resonance frequency using dumbbell DGS which first time proposed by D. Ahn. But the proposed DGS provides more satisfactory result compare to dumbbell DGS. The responses of the dumbbell DGS and proposed DGS are shown in the following figure 4.

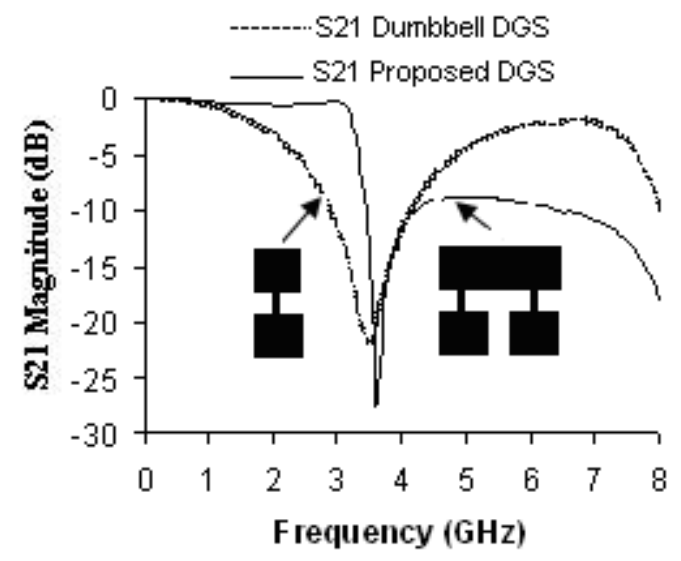

Figure 4. Comparison of Proposed DGS unit with Dumbbell DGS

There is provision to generate attenuation pole at $3.52 \mathrm{GHz}$ with Dumbbell DGS of two Square slots of $10 \mathrm{~mm}$ length, $10 \mathrm{~mm}$ breath and $0.4 \mathrm{~mm}$ width connected by one transverse slot of length $2 \mathrm{~mm}$ and width of $0.4 \mathrm{~mm}$ underneath a $50 \Omega$ microstrip line. But the proposed DGS structure provides some advantages.

From the above figure (Figure 4) it can be observed that dumbbell DGS provides attenuation pole at $3.52 \mathrm{GHz}$ and attenuation zero at $1.95 \mathrm{GHz}$ with very poor sharpness factor $(11.4 \mathrm{~dB} / \mathrm{GHz})$ where as the proposed DGS provides high sharpness factor more than $(50 \mathrm{~dB} /$ $\mathrm{GHz}$ ) in transition band i.e there is a good transition between the passband and the stop band of the proposed structure. As well as to get the same attenuation pole or resonance frequency near 3.6 GHz the Dumbbell DGS size require more than $(10 \mathrm{~mm} \times 22 \mathrm{~mm})$ where as $(10 \mathrm{~mm} \times 10 \mathrm{~mm})$ is sufficient for the proposed DGS i.e circuitry becomes more compact in size which is most important requirement in the modern microwave and millimeter wave mobile and wireless satellite communication system.

\section{DGS under Capacitively loaded Line}

The frequency characteristics of the DGS unit under microstrip line show a very sharp lowpass filtering characteristics but the insertion loss at passband is $0.8 \mathrm{~dB}$, which is not acceptable as a practical lowpass filter. To reduce the insertion loss at passband, the standard microstrip line is replased by T-shape capacitively loaded alternative transmission line. The modified part of the microstrip line provides additional capacitance and acts as a low impedance line connected with high impedance line with standard characteristics impedance 50 Ohm and suitable for the reduction of the insertion loss.

The modified structure of the proposed DGS unit is shown in the figure 5(a). For the Tstructure, patch have width, $e=1.5 \mathrm{~mm}$ with length, $b=10 \mathrm{~mm}$, and stem have width, $w 1=0.4 \mathrm{~mm}$, length g1 $=0.5 \mathrm{~mm}$, is the optimized dimensions of the above DGS unit. In the layout of DGS unit, a rectangular ring slot (of length $a=4 \mathrm{~mm}$, breadth $b=10 \mathrm{~mm}$ and width $h=0.4 \mathrm{~mm}$ ) is connected with two square ring slots (of side length $\mathrm{e}=4 \mathrm{~mm}$ and width $\mathrm{h}=0.4 \mathrm{~mm}$ ) by two thin transverse slots (of length $\mathrm{L}=6 \mathrm{~mm}$ and width $\mathrm{g}=0.4 \mathrm{~mm}$ ). The simulated S-parameters of the proposed DGS with capacitively loaded line are compared with DGS with standard 50 Ohm line as shown in Figure 5(b). From the simulated S-parameters of the DGS the 3dB cutoff frequency at $2.45 \mathrm{GHz}$ and pole frequency at $2.62 \mathrm{GHz}$ with maximum attenuation of $35 \mathrm{~dB}$ 
are observed. The passband insertion loss is $0.65 \mathrm{~dB}$ but the DGS under capacitively loaded line, the $3 \mathrm{~dB}$ cutoff frequency at $1.89 \mathrm{GHz}$ and pole frequency at $2.2 \mathrm{GHz}$ with maximum attenuation of $27.3 \mathrm{~dB}$ are observed with the passband insertion loss is $0.01 \mathrm{~dB}$ compares to 0.8 $\mathrm{dB}$ for microstrip line which makes the filter more practicable.

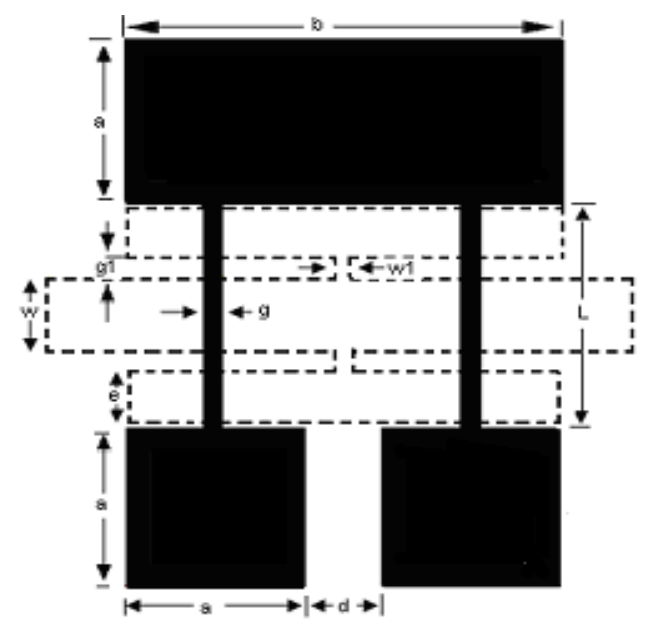

(a)

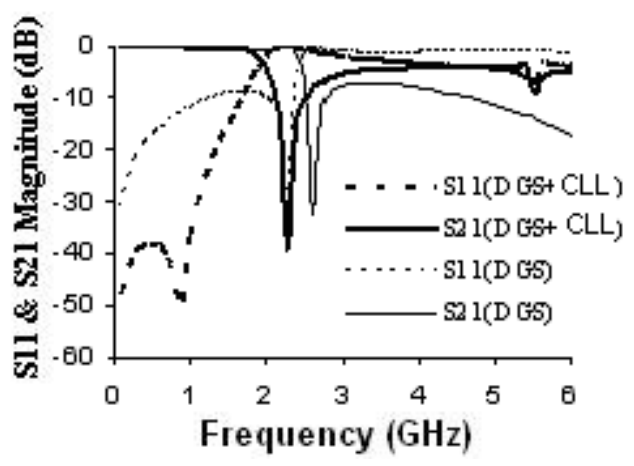

(b)

Figure 5(a). DGS unit underneath a capacitively loaded line (b) S-parameters of the DGS under standard (50 ohm) line and capacitively loaded line

Tuning of DGS with transverse slot width ' $g$ ':

The elliptic- function response can be tuned with variation the value of the transverse slot width ' $g$ '. The following table1 and Figure 6(a), 6(b), 6(c) shows the variation of different parameters with different values of transverse slot width width ' $g$ ' of the proposed DGS.

Graphical representation of the Table 1 shows as follows:

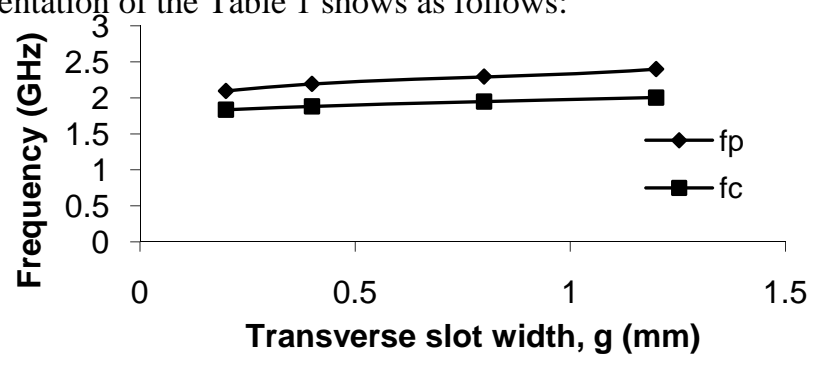

Figure 6(a). fp \& fc characteristic with transverse slot width 
Table 1. Variation of different parameters with different values of transverse slot width.

\begin{tabular}{|c|c|c|}
\hline $\begin{array}{c}\text { Transverse slot width } \\
\mathrm{g}(\mathrm{mm})\end{array}$ & $\begin{array}{c}\text { Attenuation pole freq } \mathrm{f}_{\mathrm{p}} \\
(\mathrm{GHz})\end{array}$ & $\begin{array}{c}\text { Attenuation zero freq } \mathrm{f}_{\mathrm{c}} \\
(\mathrm{GHz})\end{array}$ \\
\hline 0.2 & 2.096 & 1.836 \\
\hline 0.4 & 2.195 & 1.883 \\
\hline 0.8 & 2.2965 & 1.9502 \\
\hline 1.2 & 2.3998 & 2.0049 \\
\hline
\end{tabular}

From the above curve [Figure 6 (a)] and with the help of curve fitting it is possible to find the value of the require transverse slot width, g, of the DGS for the specified value of the attenuation pole frequency, fp, utilizing the following equation :

$$
y=0.4077 x^{3}-0.9729 x^{2}+0.9646 x+1.9387
$$

Where, $\mathrm{Y}$ and $\mathrm{X}$ signify the transverse slot width (g) and attenuation pole frequency (fp) respectively.

In the same way, from Figure 6 (a) require transverse slot width, g, can be tuned with attenuation zero ( $3 \mathrm{~dB}$ cut-off) frequency, fc, utilizing the following equation :

$$
y=0.0726 x^{3}-0.2133 x^{2}+0.3427 x+1.7754
$$

Where, $\mathrm{Y}$ and $\mathrm{X}$ signify the transverse slot width (g) and attenuation zero frequency (fc) respectively.

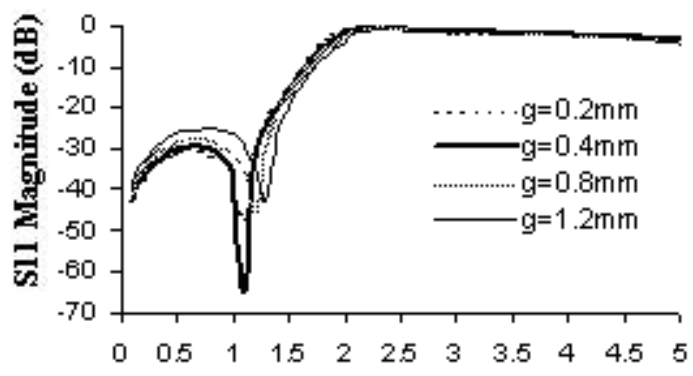

Frequency (GHz)

(b)

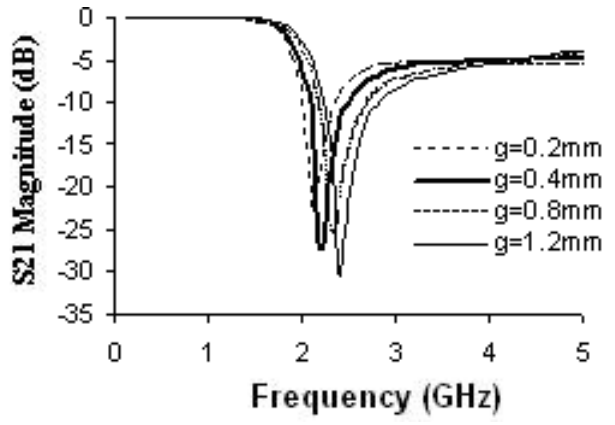

(c)

Figure 6(b). S11 \& (c) S21 parameters for different values of transverse slot width 'g' 
From the above discussion it is clear that it is possible to optimized the transverse slot width ' $g$ ' of the proposed DGS for a given fp and fc value with the help of equation (1) and (2).

\section{Tuning of DGS with transverse slot Length ' $L$ ':}

Table 2. Variation of different parameters with different values of transverse slot length:

\begin{tabular}{|c|c|c|}
\hline $\begin{array}{c}\text { Transverse slot length }, \mathrm{L} \\
(\mathrm{mm})\end{array}$ & $\begin{array}{c}\text { Attenuation pole freq } \mathrm{f}_{\mathrm{p}} \\
(\mathrm{GHz})\end{array}$ & $\begin{array}{c}\text { Attenuation zero freq } \\
\mathrm{f}_{\mathrm{c}}(\mathrm{GHz})\end{array}$ \\
\hline 4 & 2.6056 & 2.2144 \\
\hline 6 & 2.195 & 1.883 \\
\hline 8 & 1.996 & 1.7313 \\
\hline 10 & 1.7996 & 1.612 \\
\hline 12 & 1.698 & 1.518 \\
\hline
\end{tabular}

Graphical representation of the Table 2 shows as follows:

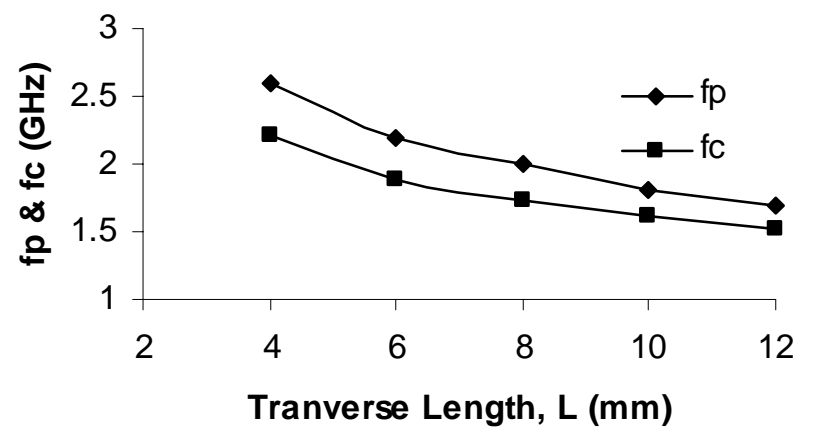

7(a) fp \& fc characteristic with transverse slot length ' $L$ '

From the above curve [Figure 7(a)] and with the help of curve fitting it is possible to find the value of the require transverse slot length ' $L$ ', of the DGS for the specified value of the attenuation pole frequency, fp, utilizing the following equation:

$$
y=0.0008 x^{4}-0.0263 x^{3}+0.3276 x^{2}-1.889 x+6.4036
$$

Where, $\mathrm{Y}$ and $\mathrm{X}$ signify the transverse slot length ' $\mathrm{L}$ ' and attenuation pole frequency (fp) respectively.

From Figure 7(a) require transverse slot length ' $L$ ', can be tuned with attenuation zero (3 $\mathrm{dB}$ cut-off) frequency, fc, utilizing the following equation :

$$
y=0.0004 x^{4}-0.0133 x^{3}+0.1814 x^{2}-1.1593 x+4.7065
$$

Where, $\mathrm{Y}$ and $\mathrm{X}$ signify the transverse slot length ' $\mathrm{L}$ ' and attenuation zero frequency (fc) respectively. 


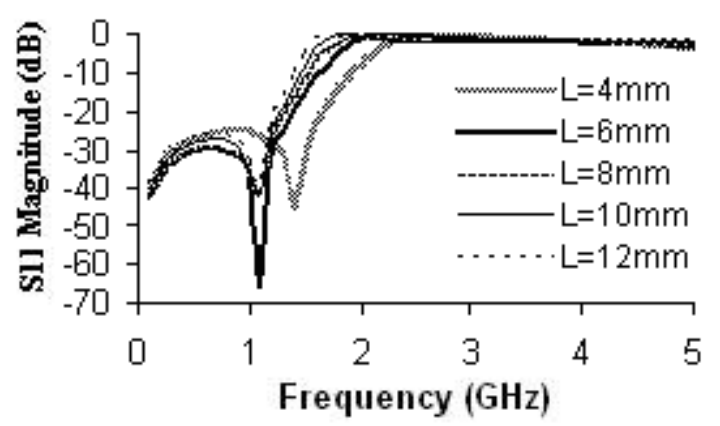

(b)

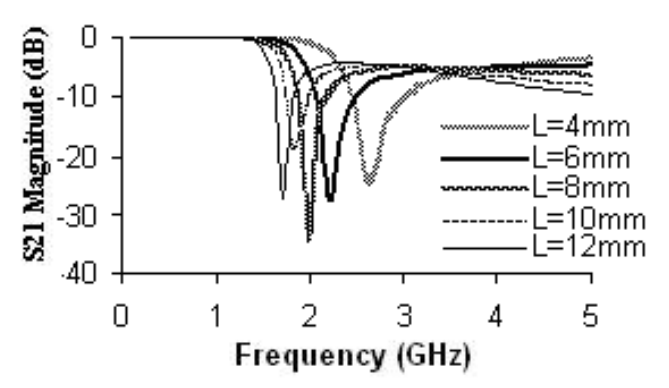

(c)

Figure 7 (b) S11 \& (c) S21 parameters for different values of transverse slot Length ' $L$ '

From the above discussion it is clear that it is possible to optimized the transverse slot Length ' $L$ ' of the proposed DGS for a given fp and fc value with the help of equation (3) and (4).

\section{Realisation of Lowpass Filter}

A lowpass filter has been realized using three DGS units of different sizes under capacitively loaded microstrip line or alternative transmission line as shown in Figure 8. The separation (x) between two DGS cell is taken as $2 \mathrm{~mm}$. The overall length of DGS filter including microstrip line is $38 \mathrm{~mm}$. The dimensions of the rectangular lengths of DGSs are $7.5 \mathrm{~mm}, 10 \mathrm{~mm}$ and $12.5 \mathrm{~mm}$ respectively. The transverse slot lengths are $8 \mathrm{~mm}, 6 \mathrm{~mm}$ and $4 \mathrm{~mm}$ respectively. The other dimensions are related with the relations of optimization as describe previously.

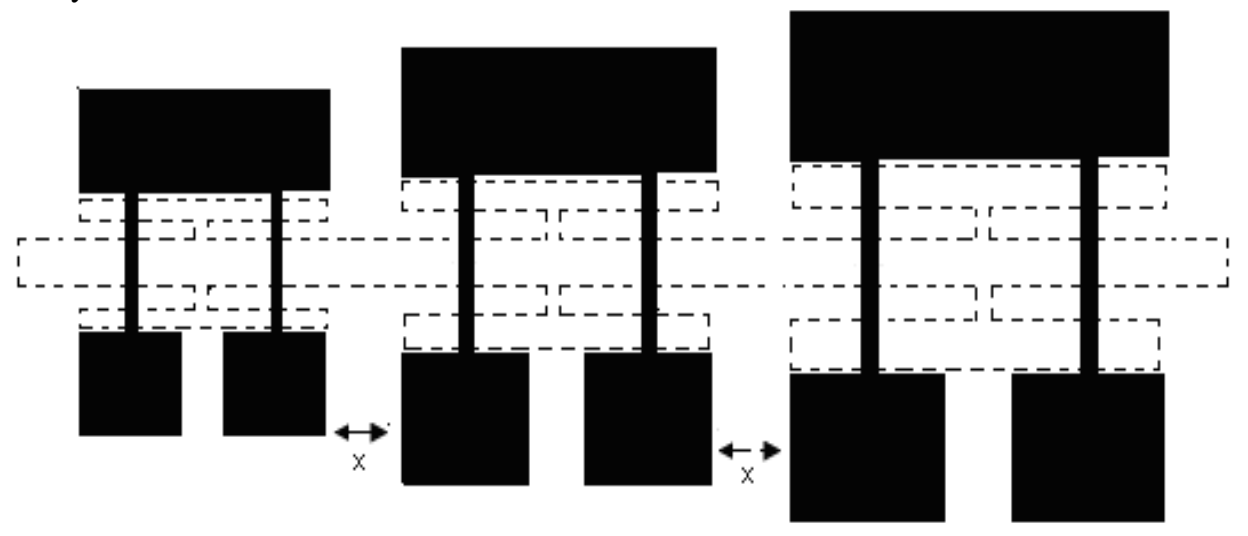

Figure 8. Layout of a DGS based lowpass filter 
The equivalent circuit is obtained by cascading the equivalent circuits of the individual DGS units with 50 ohm transmission line is illustrated in Figure 9.

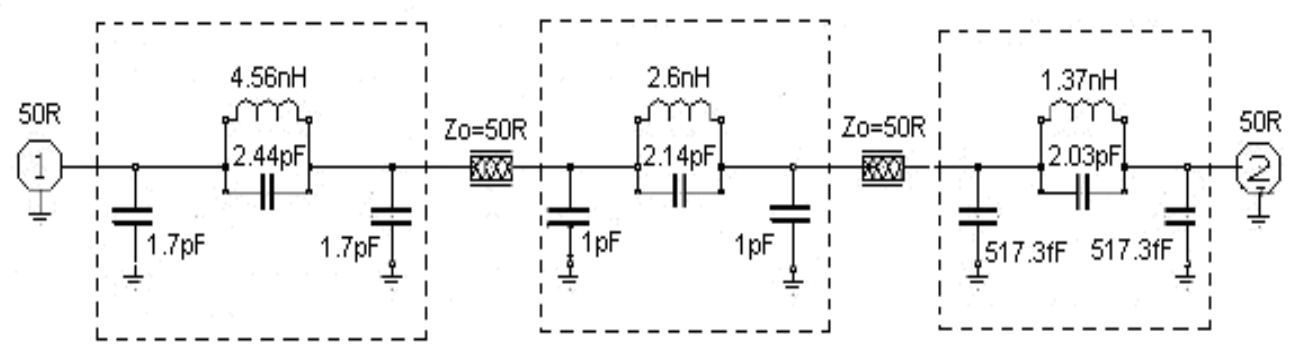

Figure 9 Equivalent Circuit

The simulation of the DGS for lowpass filter has been done with the help of IE3D EMsimulator and Circuit model analysis has been done by softwere RF sim99 and Filter Free Nuhertz technology. The simulated and circuit model responses are shown in Figure 10. It is observed that there is a good agreement between them.

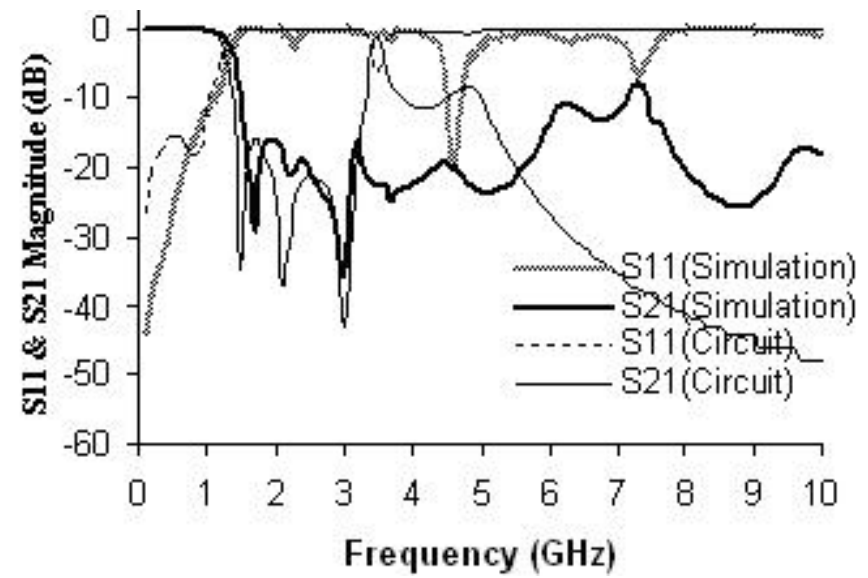

Figure10. S-parameters of DGS based low-pass filter simulated \& circuit model

The fractional bandwidth (FBW) of stopband at $-18 \mathrm{~dB}$ is 0.688 or almost $69 \%$ and the attenuation bandwidth for EM-simulated and circuit-model are $1.6 \mathrm{GHz}$ and $1.8 \mathrm{GHz}$ respectively with center frequency $2.3 \mathrm{GHz}$. The insertion loss in passband is almost $0.02 \mathrm{~dB}$ in both simulated and circuit-model results. The sharpness at transition knee is more than $90 \mathrm{~dB}$ $/ \mathrm{GHz}$ for both simulated and circuit-model results.

It responses provide the -3dB cut-off and attenuation pole frequencies at $1.38 \mathrm{GHz}$ and 1.7 $\mathrm{GHz}$ respectively in simulation and $1.41 \mathrm{GHz}$ and $1.72 \mathrm{GHz}$ in measurement results. Both responses provide $1.56 \mathrm{GHz}$ passband bandwith at $-15 \mathrm{~dB}$. The simulated and measurement response provides the sharpness factors of $93 \mathrm{~dB} / \mathrm{GHz}$ and $126 \mathrm{~dB} / \mathrm{GHz}$ respectively with almost zero passband insertion loss. Previously, from Figure10 it was found that both simulated and circuit model characterizes the almost identical responses and that is true for the simulated and measurement results as shown in Figure12. Therefore, from Figure10 and Figure12 it can conclude that there is a good agreement between simulated, circuit model and measurement results and the proposed structure is acceptable in practical applications. The passband band width can be tuned by controlling the separation between the adjacent DGS unites keeping the other dimensions constant. Thus, the structure shows a good quasi-elliptic lowpass filtering characteristics which is suitable for modern microwave and millimeter wave communication system. 
The photonic view of the proposed DGS structure is shown in the Figure11.

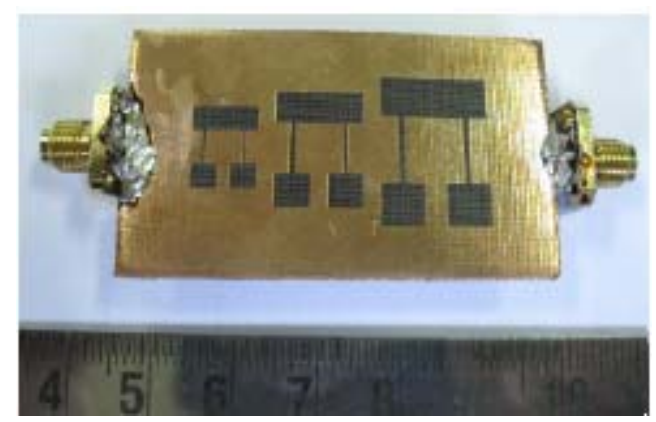

(a)

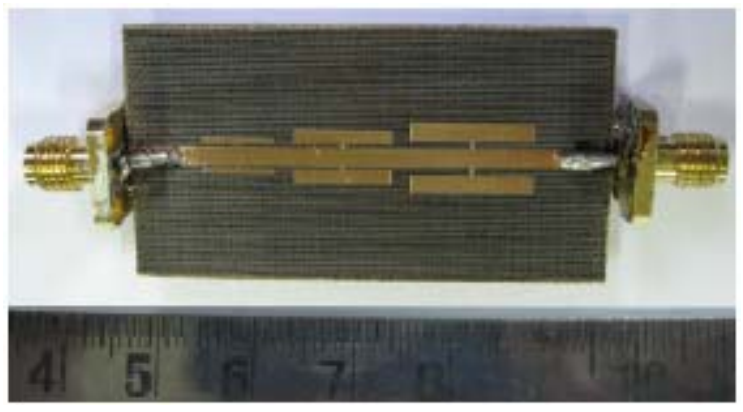

(b)

Figure 11. Photographic views of (a) ground plane (array of DGS cells) and (b) signal plane (Capacitively loaded transmission line)

The following response provides the comparative study of the simulated and measurement results.

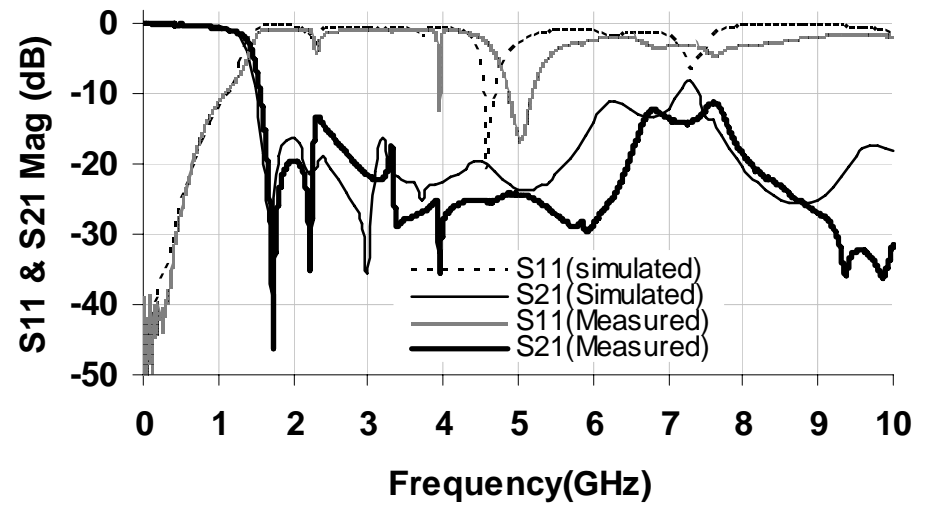

Figure 12. S-parameters of DGS based low-pass filter simulated \& measured

\section{Conclusion}

A new modified Pi shaped microstrip DGS structure is proposed. It exhibits the ellipticfunction response and almost ideal lowpass filtering characteristics. The stopband can be tuned by varying the length and width of connecting transverse slot. A considerable improvement in insertion loss has been achieved by incorporating capacitively loaded microstrip line or 
alternative transmission line above DGS. This approach leads also to a circuit size reduction. By incorporating three DGS units of different dimensions under capacitively loaded microstrip line, a three-pole LPF is realized. The stopband is tuned by changing the transverse slot width and the separation between the adjacent DGS units. A considerable improvement in steepness of the attenuation slope and fractional bandwidth has been achieved.

\section{Acknowledgment}

The work is done under the project funded by CSIR, New Delhi, Govt. of India.

\section{References}

[1] C. S. Kim, J. S. Park, D. Ahn and J. B. Lim, "A novel one dimensional periodc defected ground structure for planar circuits," IEEE Microwave and Guided wave Letters, vol. 10, No. 4, pp.131-133, 2000.

[2] D. Ahn, J. S. Park, C. S. Kim, J. Kim, Y. Qian and T. Itoh, "A design of the lowpass filter using the novel microstrip defected ground structure," IEEE Trans. on Microwave Theory and Techniques, vol. 49, no. 1, pp. 86-93, 2001.

[3] Lim J., Kim C., Lee Y., Ahn D., and Nam S., "Design of lowpass filters using defected ground structure and compensated microstrip line,” Electronics Letter, vol. 38, no. 25, pp. 1357-1358, 2002.

[4] A. Abdel-Rahman, A. K. Verma, A. Boutejdar, and A. S. Omar, "Control of bandstop response of Hi-Lo microstrip lowpass filter using slot in ground plane," IEEE Trans. On Microwave Theory Tech., vol .52, no. 3, pp. 1008-1013, March 2004.

[5] M. K. Mandal and S.Sanyal, "A novel defected ground structure for planar circuits," IEEE Microwave and Wireless Comp. Lett., vol. 16, no. 2, pp.93-95, Feb. 2004.

[6] Chen J. -X., Li J. -L., Wan K. -C. and Xue Q., "Compact quasi-elliptic function filter based on defected ground structure," IEE Proc.-Microwave Antennas propagation, vol . 153, no. 4, pp. 320-324, Aug. 2006.

[7] Susanta Kumar Parui, Santanu Das 'An asymmetric defected ground structure with elliptical response and its application as a lowpass filter’ Int. J. Electron. Commun. (AEÜ) vol. 63 ,pp.483 - 490, 2009.

[8] Parui, Susanta Kumar, Moyra, Tamasi and Das, Santanu "Quasi-elliptic filter characteristics of an asymmetric defective ground structure," International Journal of Electronics, Vol.-96, No.-9, PP-915-924. September 2009.

[9] A. Tirado-Mendez, H. Jardon-Aguilar, and R. Flores-Leal "Improving frequency response of microstrip filters using defected ground and defected microstrip structures” Progress In Electromagnetics Research C, Vol. 13, 77 - 90, 2010. 


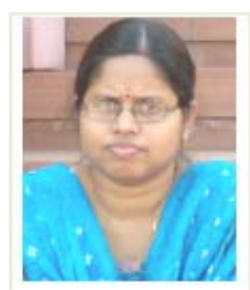

Tamasi Moyra (1978) received the AMIE (India) degree in Electronics and Communication Engineering from Institution of Engineers, India in the year 2003 and M.E. degree in the year 2007 from Bengal Engineering and Science University, Shibpur, India in the department of Electronics and Telecommunication Engineering. She is currently working toward the Ph.D degree in the department of Electronics and Telecommunication Engineering at Bengal Engineering and Science University, Shibpur, India. Presently, she is the Lecturer of College of Engineering and Management, Kolaghat. Her current research interests include the planar circuits, Microstrip filters, antenna elements etc. She is a life member of Institution of Engineers, India.

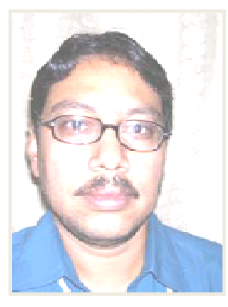

Susanta Kumar Parui (1965) received the B.Sc. degree in Physics and B.Tech. degree in Radiophysics and Electronics from University of Calcutta in the year 1987 and 1990, respectively. He has done Master degree in Microwave Communication Engineering from Bengal Engineering College, India, in the year 1993. From 1993 to 2000, he worked as Instrument Engineer. Since 2000, he is associated with the Department of Electronics and Telecommunication Engineering of Bengal Engineering and Science University, India, and presently holds the post of Senior Lecturer. His current research interests include the planar circuits, filters, antenna elements and electromagnetic band gap structures.

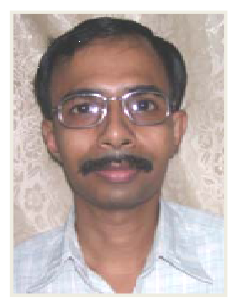

Santanu Das (1968) received the B.E.degree in the year 1989 in Electronics and Telecom. Engineering from Bengal Engineering College of Calcutta University (India) and M.E. degree in the year 1992 in Microwave Engineering from Jadavpur University, Calcutta. He obtained the Ph.D. (Engineering) degree in the year 1998 from Jadavpur University. He joined as Lecturer in the Electronics and Telecommunication Engineering Department of Bengal Engineering and Science University, India in the year 1998 and presently holds the post of Assistant Professor. His current research interests include the microstrip circuits, FSS, antenna elements and arrays. He is a life member of Institution of Engineers, India. 